\title{
Stress analysis of flexible packaging for the integration of electronic components within woven textiles
}

\author{
Menglong Li*, John Tudor, Russel Torah and Steve Beeby \\ Department of Electronics and Computer Science, University of Southampton, Southampton, UK \\ Email: m11g12@soton.ac.uk
}

\begin{abstract}
This paper presents the use of Finite Element Analysis (FEA) to model a new packaging technique capable of minimizing the impact of bending or shear stresses on components integrated within the yarn of an electronic textile. FEA has been used to model four conditions: shear load, tensile load, three point bending load and a change in temperature. Three types of adhesive (Dymax 3031, Delomonopox Mk055 and Delomonopox NU355) combined with three substrate materials, PEEK, Kapton and Mylar have been analyzed. The Kapton substrate with the Dymax 3031 adhesive are identified as the preferred material combination for the packaging assembly. The simulation results indicate that the lower Young's modulus of the adhesive and substrate materials produces smaller stresses in the shear, tensile and bending models. The lower coefficient of thermal expansion (CTE) of these materials also produces lower stresses when thermally cycled.
\end{abstract}

Keywords-Electronic textile, tensile test, FEA stress simulation, shear test, three points bending test, themal test.

\section{INTRODUCTION}

Electronic textiles (E-textiles) are a type of products with electronic components and devices embedded onto, or in fabrics; electronic textiles also are called smart fabrics [1]. E-textiles can be used in many areas, for example, clothing, furniture, medical and aerospace applications. An example of electronic textiles is wearable medical sensors to monitor the physiological health of the wearer [2]. This paper concerns the development of the materials and manufacturing methods for the fabrication of electronic circuits within a textile yarn. The electronics, comprising a silicon die (ultra-thin), sensors and electrical interconnects, will be packaged on a long thin plastic circuit that is surrounded by packing fibres and formed into a yarn. The electronic yarn should appear and feel as close as possible to a standard textile yarn.

Since an electronic textile is a wearable product subject to human motion and activities, integrated electronic components will be subject to different stresses such as bending which potentially can cause damage. Similarly, temperature changes in the environment can induce, by thermal expansion, stresses in the electronic components and adhesives which may result in failure. Therefore, the need to develop a new reliable electronic packaging method to mitigate these stresses is important.

A flip-chip electronic packaging methods, shown schematically in Figure 1, is analyzed in this paper based on the electronic die being mounted onto a plastic substrate using adhesive. The electronic die are electrically connected to each contact pad on the substrate using flexible conductive adhesive for achieved more flexibility than using solder. An additional non-conductive adhesive is added as an under-fill to increase the reliability of the electronic package, and the electronic die is completely encapsulated using a thin top plastic layer as shown in Figure 2. FEA simulation is used to evaluate the mechanical performance of different electronic packaging assemblies and minimize packaging stresses.

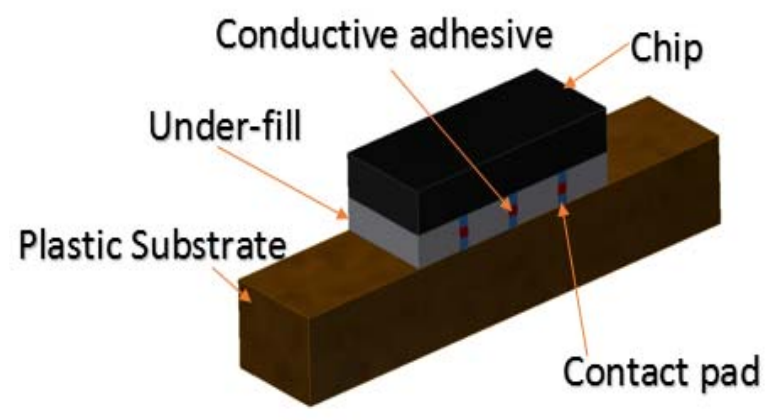

Figure 1: Flip-chip mounting of electronics on plastic substrate.

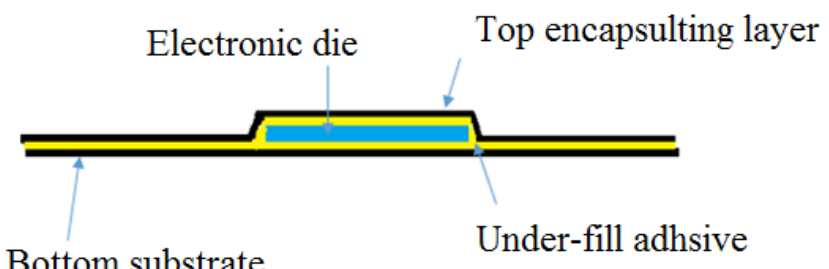

Figure 2: Cross-section of fully encapsulated electronic die covered by top and bottom films. 
This paper is divided into four sections. Section two describes the four modelling methods to simulate shear load, tensile load, thermal cycling and three points bending. The first principle stress, minimum principle stress, von-Mises stress and maximum shear stress have been analyzed in all four models as described in section three. The conclusions are presented in section four.

\section{MODELLING METHOD}

Achieving a reliable methodology for packaging bare die on thin plastic substrates requires an extensive modelling exercise to minimize stresses in the assembly that occur due to bending and temperature changes during normal operation. The packaging stresses have been modelled using ANSYS Finite Element Analysis (FEA) in a static stress analysis.

FEA has been used to determine the stresses in the mounted ultra-thin die/adhesive/plastic substrate assembly under four conditions. The four conditions include a shear load, a tensile load, a three point bending load and under a change in temperature. The models used are shown in Figures 3 and 4. These models have been used to optimize the thickness of the die, select the best adhesive and substrate materials used in the assembly to minimize the stresses.

Thermal stress is produced by temperature changes [3] in the packaged electronic device, and is potentially a key failure mechanism. A thermal expansion model is developed to simulate the stresses inside the electronic package caused by temperature changes. Figure 3(a) shows the thermal expansion model, a long substrate layer will allow the die and adhesive to bend more easily. The left edge of substrate was fixed, and the whole package is heated up from $0{ }^{\circ} \mathrm{C}$ to $100{ }^{\circ} \mathrm{C}$.

The shear test model as shown in Figure 3(b) is based upon the industrial standard test document MIL-STD-883 [4]. A force is applied to the left edge of the die and perpendicular to the primary substrate. The left and right edges of the substrate are fixed and the top and bottom surface of the substrate also are fixed.

Figure 4(a) shows the three point bending model which replicates a practical testing set-up $[5,6]$. The two stands are used in this bending test to allow space for the packaged electronic die to bend. The two stands are fixed, and an external force is applied to location " $a$ " at the center of the electronic die.

The tensile test model is also based on the industrial test standard document MIL-STD-883 [5]. In the document, section 3.1.6: test condition H- Pull-off test states that the substrate should be fixed while a pull-off stress is applied to the die [5]. Figure 4(b) shows the tensile test model. In the model, an external force is applied on the top surface of the die, and the left and right edges and top and bottom surface of the substrate are fixed.

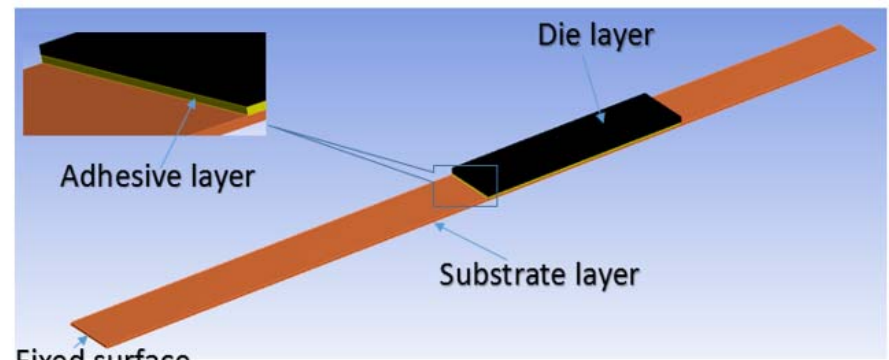

(a)

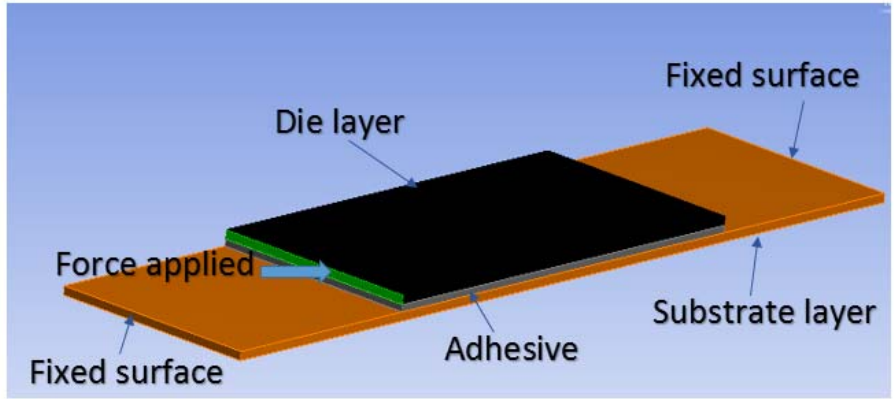

(b)

Figure 3: FEA modelling method, (a) diagram for thermal expansion model and (b) shear load model.

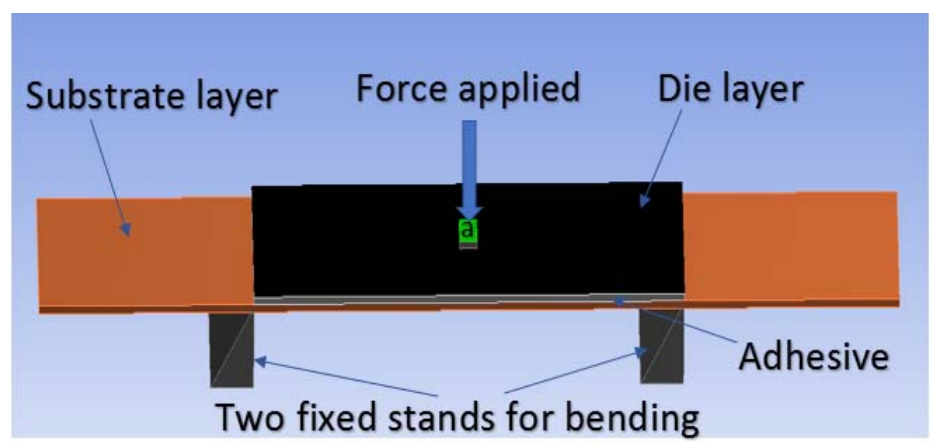

(a)

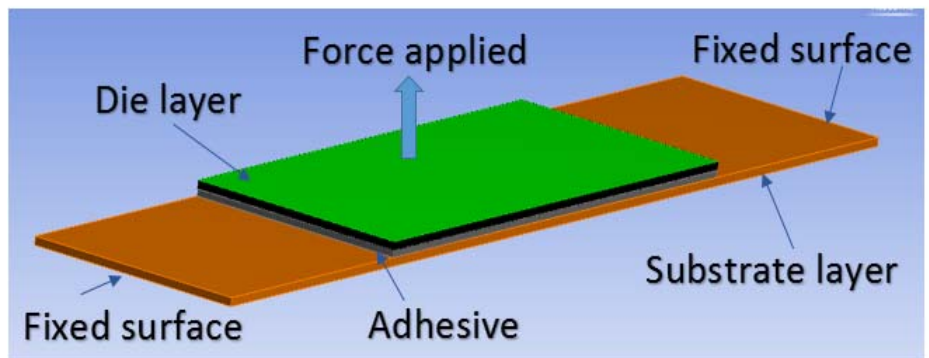

(b)

Figure 4: FEA modelling method, (a) diagram for three points bending model and (b) tensile load model. 


\section{SIMULATION RESULTS AND DISSCUSSION}

The simulations presented in this paper aim to determine how the geometric and material parameters influence on the stresses in the packaged electronic device. These parameters include the thickness of the die, the substrate, the under-fill and the materials used for the substrate and under-fill.

The stresses induced within an electronic textile contribute to the failure of the packaged electronic component; external stresses are one of the main reason for die, adhesive and substrate to fail in electronic component packaging. In reference to the three failure theories (maximum shear stress theory, maximum distortion energy theory and maximum normal stress theory) [7], the first principal stress, minimum principal stress, von-Mises stress and maximum shear stress which are caused by external force have been analyzed in the four simulation models.

\begin{tabular}{|c|c|c|c|c|}
\hline Product & $\begin{array}{c}\text { Young's } \\
\text { Modules } \\
(\mathrm{MPa})\end{array}$ & $\begin{array}{c}\mathrm{CTE} \\
\left(\mathrm{K}^{-1}\right)\end{array}$ & $\begin{array}{c}\text { Density } \\
\left(\mathrm{gcm}^{-3}\right)\end{array}$ & $\begin{array}{c}\text { Tensile } \\
\text { Strength } \\
(\mathrm{MPa})\end{array}$ \\
\hline $\begin{array}{c}\text { Silicone } \\
\text { die }\end{array}$ & 150000 & $1.1 \mathrm{E}-06$ & 2.33 & 7000 \\
\hline $\begin{array}{c}\text { Dymax } \\
3031\end{array}$ & 100 & $178 \mathrm{E}-06$ & 1.03 & 10 \\
\hline MK055 & 3200 & $6.4 \mathrm{E}-05$ & 1.2 & 50 \\
\hline NU355 & 1700 & $150 \mathrm{E}-06$ & 1.1 & 42 \\
\hline PEEK & 3800 & $4.7 \mathrm{E}-05$ & 1.32 & 98 \\
\hline Kapton & 2500 & $2 \mathrm{E}-05$ & 1.42 & 231 \\
\hline Mylar & 3100 & $1.7 \mathrm{E}-05$ & 1.39 & 138 \\
\hline
\end{tabular}

Table 1: the properties of materials

Shear stress and von-Mises stress are the most important factors for evaluating the strength of the adhesive and substrate layers of an electronic component package [7]. The first principal stress, minimum principal stress are the main stresses to electronic die failure [7]. The first principal stress is the maximum tensile stress and the minimum principal stress is the maximum compressive stress. In all simulations the first principal stress and minimum principal stress are detected in the die layer. In the simulations to compare under-fill adhesive materials, the shear stress and the von-Mises stress are detected in adhesive layer. In the substrate materials comparison simulations the von-Mises stress are detected in substrate layer. The length and width of the die and the under-fill layer are fixed at $5 \mathrm{~mm}$ and $1 \mathrm{~mm}$ respectively. The substrate has a length of $10 \mathrm{~mm}$ and a width of $1 \mathrm{~mm}$ for shear load, tensile load and the three point bending load test whilst the length of substrate increased to $20 \mathrm{~mm}$ for the thermal expansion test to allow the die and adhesive to bend more easily. Three types of adhesive (Dymax 3031, Delomonopox Mk055 and Delomonopox NU355) and three substrate materials, PEEK, Kapton and Mylar have been simulated and the properties of those material as shown table 1. In all simulations the thicknesses of the die, adhesive and substrate are $0.025 \mathrm{~mm}, 0.01 \mathrm{~mm}$ and $0.05 \mathrm{~mm}$ respectively.

\subsection{COMPARISION OF UNDER-FILL ADHESIVE MATERIAL}

The relationship between the adhesive materials and the stresses on the packaged electronic device is discussed in this section. The substrate material is Kapton and the die material is silicon. A force load of $5 \mathrm{~N}$ was used for the tensile test, three point bending test and the shear test. The adhesives Dymax3031, MK055 and NU355 have been compared, and the von-Mises stress and maximum shear stress are detected in the under-fill adhesive layer in this section.

Figure 5(a) shows the simulation result for the effect of adhesive materials in the tensile test model, this shows that adhesive material changes do not affect the compressive and tensile stresses in the die layer and the under-fill adhesive. Dymax 3031 has the best performance in the tensile test model since it showed smaller von-Mises and shear stresses in comparison with the other adhesives. This is because Dymax3031 has the smallest Young's modulus. In the shear load simulations shown in Figure 5(b), the Dymax 3031 shows a slightly smaller tensile stress and also the smallest compressive stress in the die layer. In the adhesive layer, Dymax 3031 has the smallest von-Mises and shear stresses because of the lower Young's Modulus.

In the thermal expansion model, the compressive stress in the three adhesives were almost the same as shown in Figure 6(a). The Dymax 3031 produced the smallest tensile stress in the die layer and the smallest von-Mises and shear stresses in the adhesive layer, since it has the smallest coefficient of thermal expansion (CTE) compared with other under-fill materials.

The three point bending test results shown in Figure 6(b) indicate that Dymax 3031 again results in the smallest vonMises stress and shear stress in the adhesive layer. The Dymax 3031 and NU355 produced similar compressive stress and tensile stress in the die layer, where the MK055 produced the largest compressive stress and tensile stress.

All four models indicate that, of the three different adhesives, Dymax 3031 is the preferred choice. 


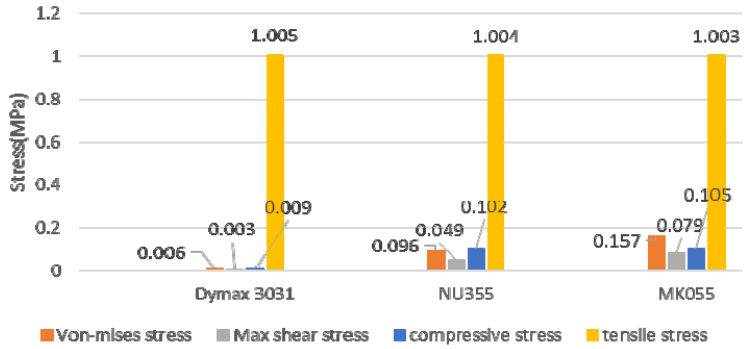

(a)

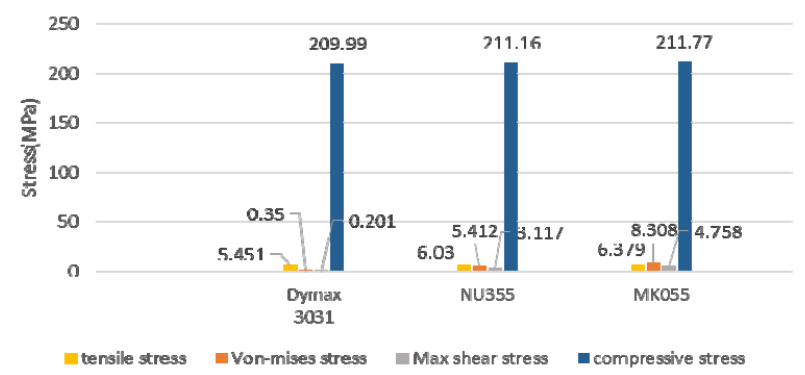

(b)

Figure 5: Simulation results comparing under-fill materials in (a) tensile load test, and (b) shear load test. (Von-mises stress and Max stress are detected in adhesive layer, tensile and compressive stress are detected in die layer.)

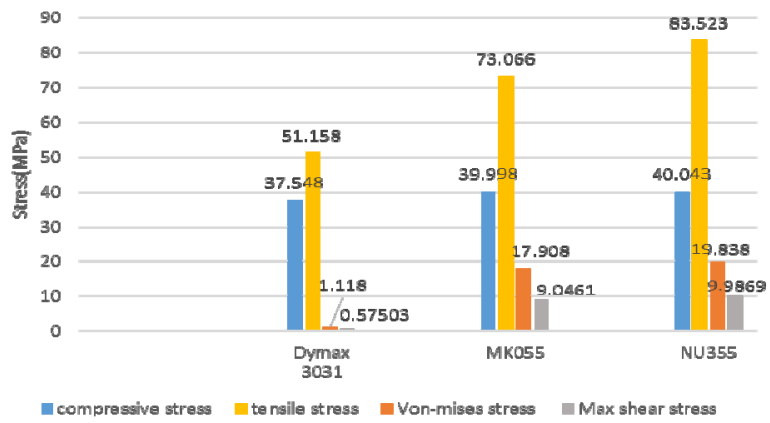

(a)

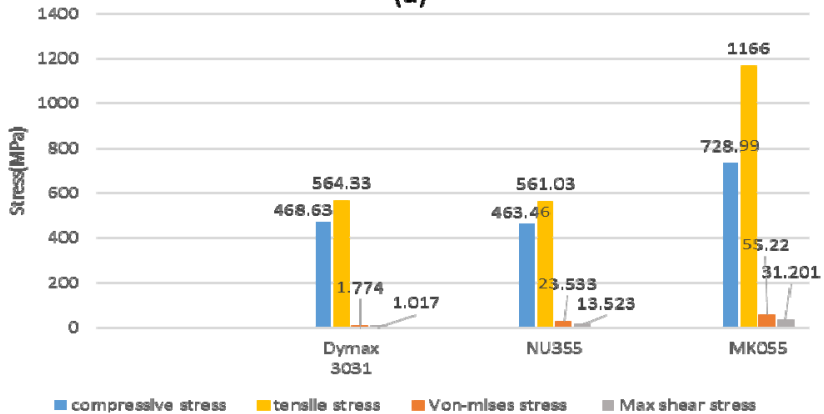

(b)

Figure 6: Simulation results comparing under-fill materials in (a) thermal expansion test, and (b) three points bending test. (Vonmises stress and Max stress are detected in adhesive layer, tensile and compressive stress are detected in die layer.)

\subsection{COMPARISION OF SUBSTRATE MATERIAL}

The choice of substrate material also affects the stresses in the packaged electronic device. In this section, PEEK, Kapton and Mylar substrates have been simulated in the four models. The die material is silicon and the adhesive is Dymax 3031. $10 \mathrm{~N}$ load is used for the three point bending, shear test and tensile models and the von-Mises stress and maximum shear stress are detected in the substrate layer for substrate comparison.

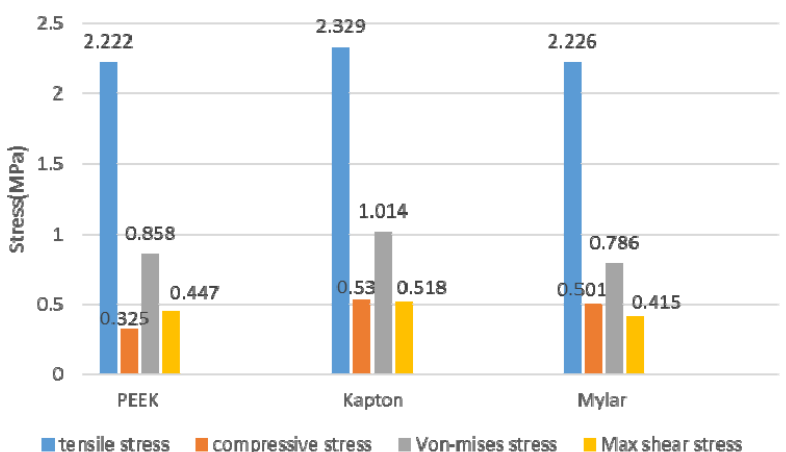

(a)

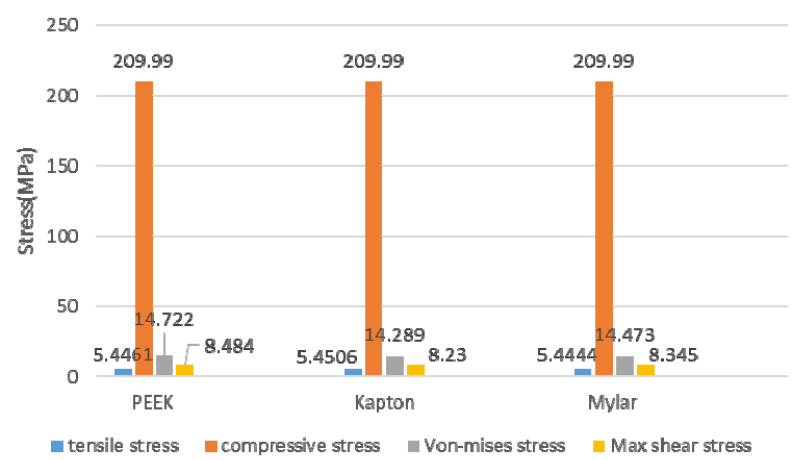

(b)

Figure 7: Simulation results comparing substrate materials in (a) tensile load test, and (b) shear load test. (Von-Mises stress and maximum shear stress are detected in the substrate layer, tensile and compressive stress are detected in die layer.)

The tensile test simulation results (shown Figure 7(a)) indicate that the von-Mises and shear stresses in the three different substrates are very small compared with the strength of substrate material. The tensile and compressive stress, detected in the die layer, also are very small compared with the strength of the die material. So, in the tensile load simulation, the use of different substrate materials should not significantly affect the failure of electronic device package. The tensile and compressive stress detected in the die layer for the shear load are also similar for the three different substrate materials, but the Kapton substrate produced the smallest Von-Mises stress and shear stress as it has the smallest Young's Modulus. 
Figure 8 shows the simulation results of the thermal expansion and bending test. The induced stresses are much lower than the die material strength, but the PEEK substrate produced the largest compressive and tensile stress. The Kapton substrate demonstrated the best performance in the substrate and die.

In the three point bending, shear load and thermal expansion models, the Kapton substrate again showed the best performance. The tensile test models also showed that the material of substrate should not significantly affect the failure of the electronic device package. Overall, Kapton should offer the highest reliability in the electronic package.

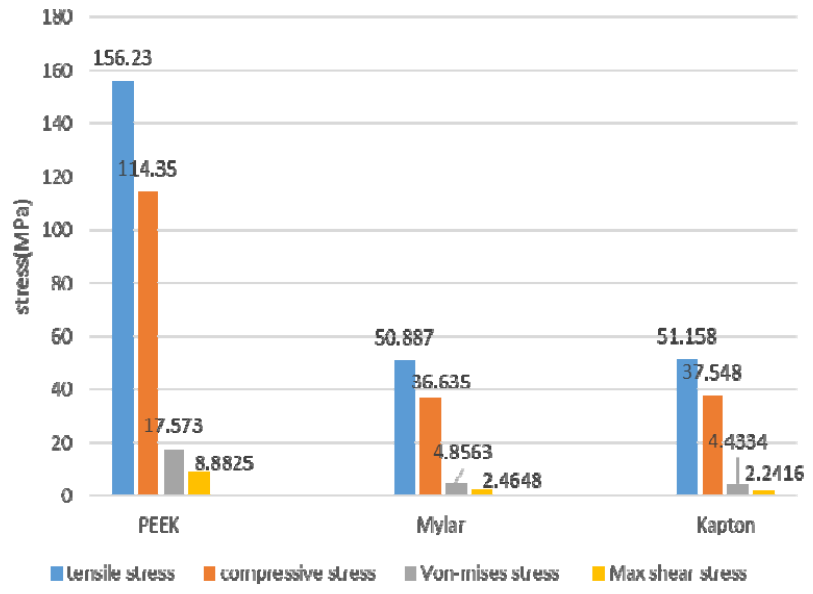

(a)

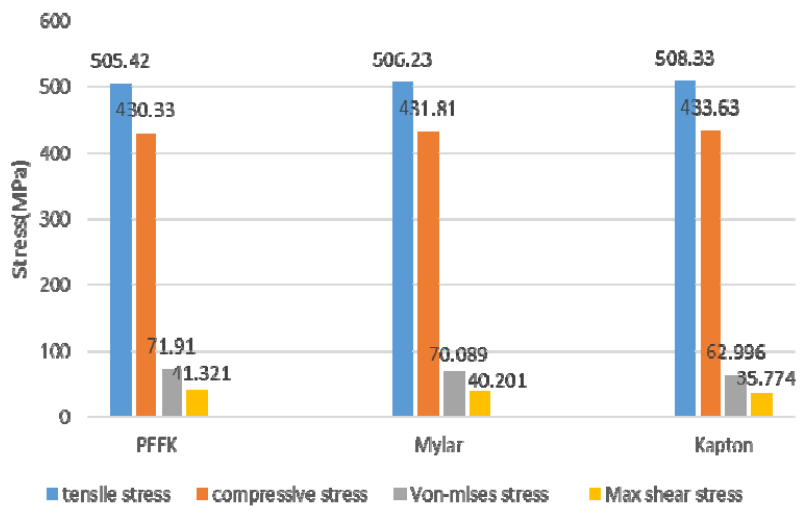

(b)

Figure 8: Simulation results for comparing substrate materials in (a) thermal expansion test, and (b) three points bending test. (VonMises stress and maximum shear stress are detected in the substrate layer, tensile and compressive stress are detected in die layer.)

\subsection{EFFECT OF ELECTRONIC DIE THICKNESS}

In order to determine the relationship between the die thickness and the stresses in the electronic device package, the thickness of the substrate and adhesive layer, and the materials used for die, adhesive and substrate all are fixed in the models. The die material used is standard silicon, the substrate material is Kapton and the adhesive layer material is Dymax 3031. The adhesive thickness is fixed to $10 \mu \mathrm{m}$ and substrate thickness is fixed at $150 \mu \mathrm{m}$. A $10 \mathrm{~N}$ loaded force is used in shear test. The bend and tensile test models have a load force of $5 \mathrm{~N}$. Die thicknesses of $0.025 \mathrm{~mm}$, $0.050 \mathrm{~mm}, 0.075 \mathrm{~mm}$ and $0.125 \mathrm{~mm}$ have been simulated and compared. The compressive and tensile stress are detected in the die layer and the von-Mises and shear stress are detected in the adhesive layer in this section.

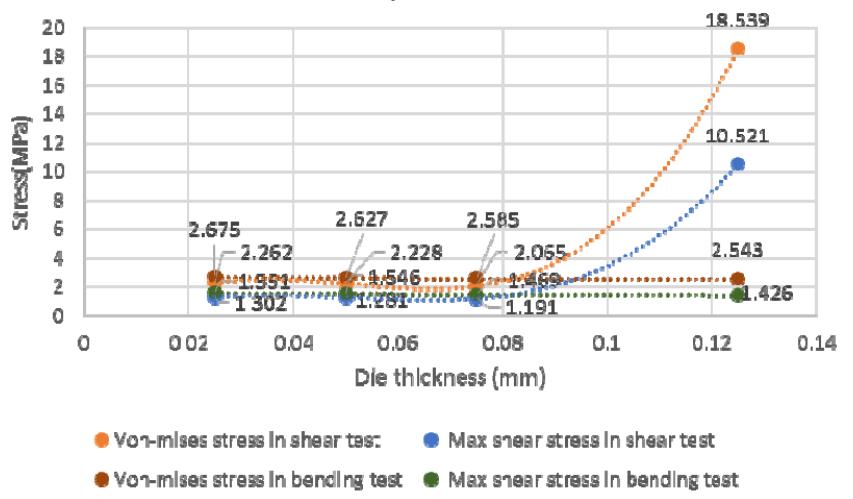

(a)

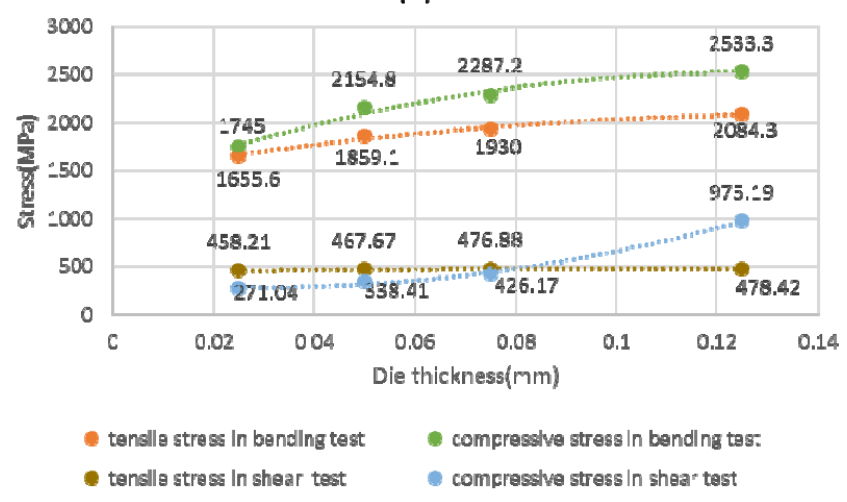

(b)

Figure 9: Simulation results for (a) shear stress and von-Mise stress in bending and shear test, (b) tensile stress and compressive stress in bending and shear test. (Von-mises stress and Max stress are detected in adhesive layer, tensile and compressive stress are detected in die layer.)

The shear stress, von-Mises stress, tensile stress and compressive stress have been compared in shear load and the three point bending test simulation as shown Figure 9. The $0.025 \mathrm{~mm}$ thick die resulted in the lowest tensile and compression stresses, although the stresses in the adhesive layer is similarly low for die thicknesses of $0.025 \mathrm{~mm}, 0.05$ $\mathrm{mm}$ and $0.075 \mathrm{~mm}$. These results indicate that thinner die result in lower packaging stresses under mechanical load.

Figure 10 shows thermal expansion and tensile load simulation results. The shear and von-Mises stresses in the 
thermal expansion and tensile models (as shown Figure 10(a)) indicate that a change of die thickness does not significantly affect the failure of adhesive. Comparing the simulation results for all four test models, the $0.025 \mathrm{~mm}$ thick die shows the best stress performance.

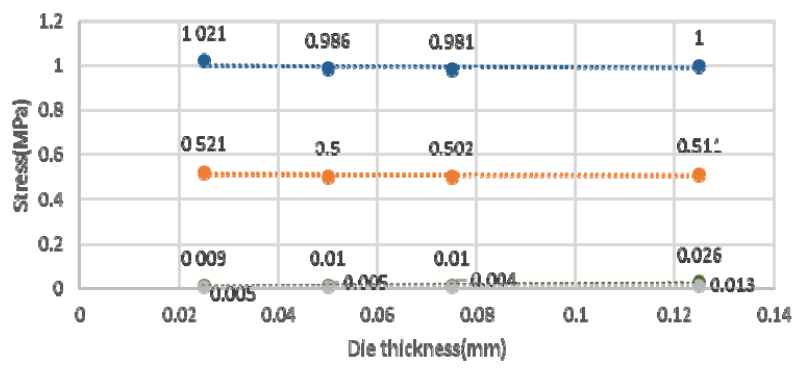

- Max shear stress In thermal expenslon test $v$ Von-mlses stress In thermal expenslon test

- Von-mlses stress in tenslla test

Max shear stress in tenslla test

(a)

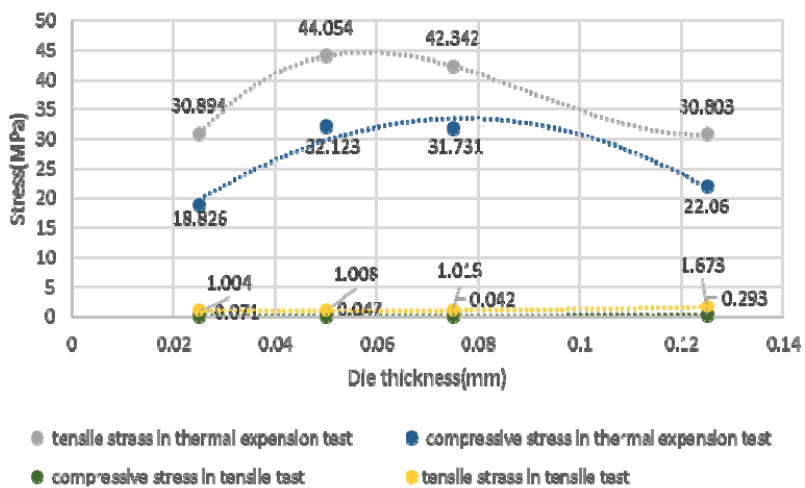

(b)

Figure 10: Simulation results for (a) shear stress and von-Mise stress in tensile and thermal expansion test, (b) tensile stress and compressive stress in tensile and thermal expansion test. (Von-Mises stress and Max stress are detected in adhesive layer, tensile and compressive stress are detected in die layer.)

\section{Conculsions}

Three types of adhesive (Dymax3031, Mk055 and NU355) and three substrate materials (PEEK, Kapton and Mylar) have been analyzed. The Kapton substrate together with the Dymax 3031 adhesive was identified as the best combination for packaging the die. The simulation results indicate that the lower Young's Modulus of the materials produces smaller von-Mises and shear stresses in the shear and bend models. The lower coefficient of thermal expansion (CTE) of these materials also produces lower von-Mises and shear stresses in the thermal simulation. The simulations identified the optimum thickness of die is 0.025 $\mathrm{mm}$.

\section{ACKNOWLEDGMENT}

This research funded by Engineering and Physical Sciences Research Council (EPSRC) project "Novel manufacturing methods for functional electronic textiles".

\section{REFERENCE}

1. Marculescu, D., et al., <Electronic textiles: a platform for pervasive computing $>$. Proceedings of the IEEE, 2003. 91(12): p. 1995-2018.

2. Windmiller, J.R. and J. Wang, <Wearable Electrochemical Sensors and Biosensors: A Review >. Electroanalysis, 2013. 25(1): p. 29-46.

3. Nakajo, A., et al., <Modeling of thermal stresses and probability of survival of tubular SOFC $>$. Journal of Power Sources, 2006. 158(1): p. 287294.

4. Department of Defense and USA, <Test method standard microcircuits $>$. MIL-STD-883E, December 1996.

5. Lai, Y.-S., et al., <Cyclic bending reliability of wafer-level chip-scale packages $>$. Microelectronics Reliability, 2007. 47(1): p. 111117.

6. Ilho, K. and L. Soon-Bok, $<$ Reliability and Failure Analysis of Lead-Free Solder Joints for PBGA Package Under a Cyclic Bending Load>. IEEE Transactions on Components and Packaging Technologies, 2008. 31(2): p. 478-484.

7. Sengupta, P., <Working Stress and Failure Theories A Simplified Approach>. MET 301: Theories of failure. 